\title{
Highly Oriented Carbon Fiber-Polymer Composites via Additive Manufacturing
}

Halil L. Tekinalp ${ }^{\mathrm{a}}$, Vlastimil Kunc ${ }^{\mathrm{b}}$, Gregorio M. Velez-Garcia ${ }^{\mathrm{a}}$, Chad E. Duty ${ }^{\mathrm{b}}$, Lonnie J. Love ${ }^{\mathrm{b}}$, Amit K. Naskar ${ }^{\mathrm{a}}$, Craig A. Blue ${ }^{\mathrm{b}}$, Soydan Ozcan ${ }^{\mathrm{a} *}$

${ }^{a}$ Material Science and Technology Division, Oak Ridge National Laboratory, 1 Bethel Valley Rd, Oak Ridge, TN 37830

${ }^{b}$ Manufacturing Demonstration Facility, NTRC II, Oak Ridge National Laboratory, 2360 Cherahala Blvd, Knoxville, TN 37932

\section{Abstract}

Additive manufacturing is distinguished from traditional manufacturing techniques such as casting and machining by its ability to handle complex shapes with great design flexibility and without the typical waste. Although this technique has been mainly used for rapid prototyping, interest is growing in direct manufacture of actual parts. For wide spread application of 3D additive manufacturing, both techniques and feedstock materials require improvements to meet the mechanical requirements of load-bearing components. Here, we investigated short fiber $(0.2$ $\mathrm{mm}$ to $0.4 \mathrm{~mm}$ ) reinforced acrylonitrile-butadiene-styrene composites as a feedstock for 3Dprinting in terms of their processibility, microstructure and mechanical performance. The additive components are also compared with traditional compression molded composites. The tensile strength and modulus of 3D-printed samples increased $\sim 115 \%$ and $\sim 700 \%$, respectively. 3D-printing yielded samples with very high fiber orientation in the printing direction (up to 91.5 \%), whereas, compression molding process yielded samples with significantly lower fiber orientation. Microstructure-mechanical property relationships revealed that although a relatively high porosity is observed in 3D-printed composites as compared to those produced by the conventional compression molding technique, they both exhibited comparable tensile strength and modulus. This phenomenon is explained based on the changes in fiber orientation, dispersion and void formation. 
Keywords: A. Carbon fibers; A. Short-fiber composites; A. Polymer-matrix composites; B. Mechanical Properties; E. Extrusion.

\section{Introduction}

Rapid prototyping (RP) is a technology in which a part can be built layer by layer to a desired geometry based on a computer-aided design (CAD) model. With RP, complex parts can easily be built in reasonable timeframes [1-3]. Therefore, use of this technology as a manufacturing process along with conventional manufacturing techniques can significantly improve and boost the manufacturing industry.

Fused deposition modeling (FDM), a leading RP technique, accomplishes the layer-by-layer build by depositing a material extruded through a nozzle in a raster pattern (i.e., in a pattern that is composed of parallel lines) in each layer $[1,2,4,5]$. However, because only a limited number of materials, such as thermoplastics and some engineering plastics, have been used as a feedstock for FDM, the final products have limited mechanical properties [6,7]. Therefore, to render this technology suitable for producing functional, load-bearing parts, FDM protocols are needed for materials development and for the manufacturing of composite products.

Fiber reinforcement can significantly enhance the properties of resins/polymeric matrix materials [8-11]. Although continuous fiber composites offer high mechanical performance, their processing is not commonplace. More commonly used for traditional low-cost composite part fabrication are the short fiber-reinforced polymers (SFRPs) with moderately improved mechanical properties $[3,12,13,14]$. SFRPs are typically produced by extrusion compounding and injection molding processes [15-20]. The mechanical properties of these SFRPs depend significantly on the fiber length distribution and fiber orientation distribution of the final parts 
[3,14,21]. During processing, fiber breakage occurs [3], affecting the mechanical properties of the final composite part. As fiber loading increases, the fiber breakage, due to increased fiberfiber interaction $[15,22,23]$, increases. Fiber breakage during processing also arises from the interaction of fibers with polymers, and processing equipment surfaces [3]. Therefore, the matrix material, the process conditions, and the fiber loading determine the final fiber length distribution of the composite. Similarly, fiber orientation distribution and void fraction of final SFRPs are also affected by the aforementioned factors.

Only a few studies report FDM of fiber-reinforced feedstock. Among these, Gray IV et al. [4] added thermotropic liquid crystalline polymer fibrils into polypropylene in order to prepare a composite feedstock for FDM. A capillary rheometer was used to simulate the FDM process, and, subsequently, the tensile properties of the extruded strands were measured. Zhong et al. [2] studied FDM processing of short glass fiber-reinforced acrylonitrile-butadiene-styrene (ABS) resin. Additions of plasticizer and compatibilizer improved feedstock processibility. Shofner et al. [6] investigated the effect of vapor-grown carbon fibers into ABS as an FDM feedstock. An average of 39\% increase in tensile strength was observed at $10 \mathrm{wt} \%$ loading of nanofiber. To the best of our knowledge, FDM processing of 5-7 $\mu \mathrm{m}$ diameter short carbon fiber-reinforced resin has not been reported, despite its high potential to reach desired mechanical, electrical and thermal properties, and low density [24-26]. Thermoplastic matrix composites further provide improved toughness and recyclability $[25,27]$.

In this study, carbon fiber-ABS composite was successfully prepared and used as an FDM feedstock. Short carbon fiber-reinforced ABS composites at different fiber loadings were prepared by both compression molding $(\mathrm{CM})$ and FDM to assess the strengths and weaknesses of the FDM process (in comparison with the more conventional CM process). Effects of the process 
and fiber loading on void formation, average fiber length, and fiber orientation distribution, and eventually their effect on the tensile strength and modulus of the final printed product, were investigated.

\section{Experimental}

\subsection{Materials and processing}

ABS copolymer (GP35-ABS-NT) was obtained from M Holland Co., IL. Chopped Hexcel AS4 carbon fibers (CF) with epoxy-based sizing of $3.2 \mathrm{~mm}$ length were obtained from $\mathrm{E} \& \mathrm{~L}$ Enterprises Inc., TN.

The carbon fibers and ABS resin were compounded with a Brabender Intelli-Torque PlastiCorder prep-mixer at $220^{\circ} \mathrm{C}$ and $60 \mathrm{rpm}$ rotor speed until the torque reading became constant. Mixtures of 10, 20,30, and $40 \mathrm{wt} \% \mathrm{CF}$ were prepared. A neat $\mathrm{ABS}$ resin was also run through the mixer at the same conditions as the control. Approximate mixing time was 13 min, including the feeding time. Next, these mixes were extruded as preforms at $220^{\circ} \mathrm{C}$ using a plunger type batch extrusion unit. For CM preforms, a slit-shaped die and for FDM printing preforms (i.e., filament), a cylindrical die of $1.75 \mathrm{~mm}$ diameter were used. During the process, the barrel temperature ranged between 220 and $235^{\circ} \mathrm{C}$.

FDM dog-bones were prepared by feeding the extruded filaments into a commercial desktop FDM unit (Solidoodle 3 from Solidoodle Co., NY) and printing. ASTM D638 type-V dog-bone dimensions were followed [28]. During printing, nozzle temperature was maintained at $205^{\circ} \mathrm{C}$, while printer table temperature was $85^{\circ} \mathrm{C}$. The layer height was set to $0.2 \mathrm{~mm}$ with the deposition direction being parallel to the loading direction in the gage section. The nozzle diameter of the FDM unit was $0.5 \mathrm{~mm}$ and the radius of curvature at the corners of the dog-bones 
was around $1.2 \mathrm{~mm}$. The printed dog-bones were precise and no post-processing machining was required/performed. Although all samples up to $30 \mathrm{wt} \% \mathrm{CF}$ were printed successfully, only several layers of the $40 \mathrm{wt} \% \mathrm{CF}$ samples could be printed owing to nozzle clogging. Thus, the reader should note that the results for this sample were only included for completeness.

For the preparation of the CM dog-bones, slit-extruded preforms were cut into shorter pieces to fit the mold, and they were compression molded at $220^{\circ} \mathrm{C}$ based on ASTM standard D4703 [29] to make rectangular bars. Next, dog-bones (ASTM D638 type-V) were cut from these bars by use of a Tensilkut template (special template for ASTM D638 Type V, Sieburg International Inc., TN), and a router (Tensilkut 10-21, serial No. 100590, Sieburg International Inc., TN).

\subsection{Testing and analysis}

The tensile properties of the CM and FDM samples were determined by testing at least five dogbone samples of each composition, performing displacement-controlled tensile tests in a servohydraulic testing machine at a strain rate of $0.0254 \mathrm{~mm} / \mathrm{s}$. A $12.5 \mathrm{~mm}$ gage-length extensometer was used for strain measurements.

Fibers were extracted from dog-bone samples using acetone. A small portion of each extracted sample was transferred onto a glass petri dish, and the acetone was allowed to evaporate. Images of the extracted fibers were taken at 20x magnification, and fiber length distributions from these images were obtained using a code developed in our laboratories. Mostly, around 1000 fibers were measured in order to obtain reliable fiber length data.

A piece from a dog-bone representing each composition was cut and mounted in epoxy. Next, these samples were polished for imaging clarity. After taking images of the polished surfaces for void fraction analysis, the surface was plasma etched to reveal the fiber orientation for imaging. 
Afterwards, a technique developed by Velez-Garcia and automated by Kunc [30] was used to calculate fiber orientation. The images were taken from the regions of the samples that were most representative of the gauge region of the dog-bones.

Fracture surfaces of the tested dog-bones were first sputter-coated with carbon. Next, SEM micrographs of the fracture surfaces were taken with a Hitachi S4800 FEG-SEM at an acceleration voltage of $5 \mathrm{kV}$ and an emission current of $20 \mu \mathrm{A}$.

\section{Results and Discussions}

The purpose of this research was to understand challenges and opportunities of fiber-reinforced composites made by 3D printing and to specifically evaluate the potential for load-bearing components. Our results show that composites with highly dispersed and highly oriented carbon fibers can be printed by FDM process as illustrated in Fig. 1. Both tensile strength and modulus increased dramatically reaching a specific strength $(52.9 \mathrm{kN} . \mathrm{m} / \mathrm{kg})$ higher than Aluminum 6061 $0(45.9 \mathrm{kN} . \mathrm{m} / \mathrm{kg})$. Detailed results are given in the following sections.

\subsection{Effect of process and fiber loading on void formation, fiber length distribution, and fiber orientation}

Fiber length, fiber orientation, and porosity affect the mechanical properties of composites. In this section, the effects of processing method (i.e., FDM vs. CM) and fiber loading on composite morphology (i.e., porosity, fiber length, and fiber orientation in the specimens) were discussed.

\subsubsection{Void formation}

The CM samples exhibit no visible void content; however, the FDM samples show significant pore formation. SEM images of the fracture surfaces and micrographs of the polished surfaces of 
the dog-bone samples are shown in Figs. 2 and 3. To understand the void formation mechanism, a closer look at the FDM process is needed. Fig. 2 a and $\mathbf{b}$ shows the porosity in the printed neat-ABS sample, which of course has no fiber effect. The porosity in this sample consists of relatively large triangular voids that are similarly oriented. These voids are mainly the gaps between the beads deposited during printing. Although the nozzle used to extrude the molten material is circular, during deposition, the bead is pressed down to a $0.2 \mathrm{~mm}$ thickness and becomes elliptical. Because the bead is still soft while being deposited, the bottom part flattens; however, the top part cools to form round edges before another bead/layer is deposited on top of it. For this reason these triangular gaps are only directed downwards. These triangular gaps (i.e., inter-bead pores) are actually channels aligned in the direction of loading, and they are not expected to significantly affect the mechanical performance of the samples. Note that there are no voids in the FDM neat-ABS sample surface other than these triangular gaps between the beads as shown in Fig. 2a and b. However, with the addition of carbon fibers into the feedstock, internal voids inside the beads (i.e., inner-bead pores) begin to form (Figs. 2c and 3f-h). Because voids inside the beads can create stress concentration points, they cause the samples to fail at lower stresses.

As shown in Fig. 3e-h, the average size of the triangular channels between the beads decreases with the presence of carbon fibers as compared to the FDM neat-ABS sample. This phenomenon can be attributed to the decrease in die-swell and the increase in thermal conductivity with carbon fiber addition. Even $10 \mathrm{wt} \%$ carbon fiber addition significantly eliminates die-swell, resulting in smaller beads and, therefore, smaller inter-bead gaps. Also, higher thermal conductivity helps the already cooled bottom beads to again soften once in contact with a hot bead deposited on top of it, leading to the improved packing and smaller gaps seen in Fig. $\mathbf{3 f}-\mathbf{h}$. 
On the other hand, brief image analysis of the polished gauge section of the fiber-reinforced FDM-printed dog-bones showed that the void volume fraction fluctuates between $16 \%$ and $27 \%$ independent of fiber content. These fluctuations in void volume can be attributed to the competing effects of changes in large voids among the beads and changes in smaller voids inside the beads (i.e., inter-bead porosity vs. inner-bead porosity), with increasing fiber content.

As explained earlier, increasing fiber content leads to better packing of the deposited beads and thus smaller inter-bead voids, whereas the increased number of fiber ends [3] is expected to cause more inner-bead void formation during printing. SEM micrographs of the fracture surfaces of the $10 \mathrm{wt} \%$ CF-loaded CM and FDM samples (see Fig. 2c and d) also show pore enlargement around the fibers in the FDM sample but not in the CM sample. This enlargement around the fibers also results in lower fiber-matrix interfacial contact area, and thus weaker fiber bonding to matrix, causing a decrease in the mechanical performance of the final composite part. We believe that inner-bead pores are created at the edge of or around the fibers, because the two phases (i.e., polymer and fiber phases) flow partially independently during the extrusion process of FDM. Therefore, improving the attachment and compatibility of two phases via surface treatment of fibers or using suitable sizing prior to FDM processing may help minimize the formation of inner-bead porosity.

\subsubsection{Fiber length distribution}

Fiber length distributions (FLD) of both CM and FDM samples are given in Fig. 4a and b. For easier comparison, weight average fiber lengths were also plotted and given in Fig. 4c. Although FLDs of CM and FDM samples were slightly different, they both followed a similar trend, and the average fiber length decreased with increasing fiber loading in composites made by both processes (Fig. 4). Even though $3.2 \mathrm{~mm}$ long fibers were used for reinforcement, during 
processing (especially, during high-shear mixing) significant fiber breakage occurred and composites with $0.4 \mathrm{~mm}$ or less average fiber length were obtained. It has been reported that during compounding/mixing of fibers with resin, dramatic fiber breakage occurs from the interactions between fibers and i) instrument surfaces, ii) resin, and iii) other fibers [3]. As the fiber content increases, the interaction between fibers increases, leading to more fiber breakage and thus to shorter fibers. The majority of the fiber breakage occurs during high-shear mixing. Since the preform extrusion step is similar for both the FDM and CM processes, both processes are expected to yield composites with similar average fiber lengths (with respect to the initial fiber length of $3.2 \mathrm{~mm}$ ).

During the CM process, no significant shear is applied to materials, so no further fiber breakage is expected. However, in the FDM process, molten material is pushed through a $0.5 \mathrm{~mm}$ nozzle and pressed down at about a $90^{\circ}$ angle, which could cause further fiber breakage. Therefore, FDM samples are expected to have a lower average fiber length at the same fiber loading.

\subsubsection{Fiber orientation distribution}

The method used by Bay and Tucker [31] was followed to characterize the samples' fiber orientation. Fiber orientation measurements were performed on 2D images of the polished surface of each sample, and the second-order orientation tensor, $\mathrm{a}_{\mathrm{ij}}$, was measured. Components of second-order orientation tensors for each sample are given in Table $\mathbf{1 .}$

Components $\mathrm{a}_{11}, \mathrm{a}_{22}$, and $\mathrm{a}_{33}$ show orientation in the direction of $\mathrm{x}_{1}, \mathrm{x}_{2}$, and $\mathrm{x}_{3}$, respectively (see Fig. 5). The results in Table 1 clearly show a characteristic difference between FDM and CM samples, but samples prepared by the same method are quite similar. The dominant orientation tensor components for $\mathrm{CM}$ samples are $\mathrm{a}_{33}$ and $\mathrm{a}_{11}$, with the former being larger. This conveys 
that fibers are mainly oriented in the $\mathrm{x}_{3}$-direction (i.e., the load-bearing direction) and the $\mathrm{x}_{1}$ direction. A closer look at preform preparation and the CM method easily explains these results. In this case, preforms are prepared by extruding the molten fiber-ABS mixture through a slitshaped die, during which fibers are oriented in the extrusion direction. Next, long pieces cut from this preform at pre-calculated weight are placed into the mold and compressed. Because these pieces do not fit perfectly into the mold, once molten and pressed, the material flows in $\mathrm{x}$ - and zdirections.

In contrast, the dominant component of the orientation tensor for FDM samples is only $\mathrm{a}_{33}$, and its nearly 1.0 value indicates that practically all fibers are oriented in the $\mathrm{x}_{3}$-direction. The $\mathrm{a}_{23}$ component being a little over 0.1 shows that fibers are slightly tilted in the $\mathrm{x}_{2}$-direction, probably because the depositing nozzle was perpendicular (i.e., in the $\mathrm{x}_{2}$-direction) to the printing direction.

From a mechanical performance standpoint, orientation in the $\mathrm{x}_{3}$-direction is of most interest because it is the load-bearing direction and, as explained above, fiber orientation in the $\mathrm{x}_{3}$ direction is dramatically higher (approaching maximum) in printed samples than in compressed samples. These results emphasize the inherent characteristic of gaining high orientation by use of the FDM process. Owing to its nature, the FDM process produces samples not only with higher fiber orientation, but also with higher molecular orientation in thermoplastic compared with more conventional processes such as $\mathrm{CM}$ and injection molding.

\subsection{Tensile properties}

Because the samples were exposed to similar thermal cycles in both processes, among the parameters that affect the mechanical properties, this study focused on fiber length distribution, 
fiber orientation, and porosity. While an increase in fiber length and fiber orientation positively affects the tensile properties, an increase in void fraction negatively affects the strength of a composite by both creating stress concentration points and lowering the fiber-matrix interface and bonding. Tensile strength and modulus measurements of dog-bone specimens prepared by both methods are shown in Fig. 6. The results show that tensile strength increases with increasing fiber content in both processes. It was observed that the neat-ABS samples prepared by the FDM process have higher tensile strength than the ones prepared by CM. At least five samples were tested for each case, and the standard deviation was insignificant (Fig. 6a), supporting the validity of this conclusion. The higher strength of the printed samples despite all the large gaps between the beads shows that the FDM process increases the molecular orientation of the polymer chains, increasing the tensile properties. A similar conclusion was also reported by Sood et al. [1].

The standard deviations in tensile strength measurements for the FDM samples were significantly lower than those for the CM samples. This result suggests that the FDM process not only increases the orientation of the polymer molecules, but also improves fiber dispersion and uniformity as the parts are manufactured point by point, layer by layer. As mentioned above, the standard deviation for neat polymers, even for the compression-molded one, is nearly zero. Thus, the increase in standard deviation with the inclusion of fibers probably arises from sample-tosample differences in fiber distribution.

Although for neat-polymer materials the FDM-printed dog-bones were stronger than the CM ones, with the addition of fibers into the system, the CM samples started to perform better. Since the fiber length distributions of samples prepared by both processes are similar, to understand the differences in strength, the competing effects of fiber orientation and void fraction must be 
compared. As shown in the previous results, fiber orientation is significantly higher for FDM samples, by which FDM samples can compensate the negative effect of porosity/weak fiber bonding and can still reach strength values close to $\mathrm{CM}$ samples The increase in tensile strength with the increase in fiber content becomes less prominent at higher fiber loadings (Fig. 6a) in both processes. This can be attributed to the decrease in average fiber length (Fig. 4) with increasing fiber content, while the increase in the number of inner voids (section 3.1.1) can explain the earlier drop in the strength increase of FDM samples. Therefore, modification/optimization of the mixing process to minimize fiber breakage, and modification of the FDM process to minimize inner-pore formation, may lead us to much stronger composite parts. Also, as shown in SEM micrographs of fracture surfaces after tensile testing (Fig. 3), the fibers had pulled out of the matrix in both FDM and CM samples, showing weak fiber-polymer interfacial adhesion, which also negatively affects composite strength. Similar to increasing average fiber length, improving interfacial adhesion can also have a significant impact on the mechanical performance of FDM-printed parts. There are many studies on improvement of interfacial adhesion in composites via modification of the fiber surface $[10,32,33]$.

Fig. $6 \mathbf{b}$ shows the Young's modulus measurements of all samples. Unlike tensile strength, the moduli of FDM and CM samples basically overlap and increase almost linearly with increasing fiber content. The modulus value of the CM composite is increased by nearly an order of magnitude at $40 \mathrm{wt} \%$ fiber loading. However, at this high loading ( $40 \mathrm{wt} \% \mathrm{CF}$ ) the FDM sample was difficult to fabricate owing to repeated nozzle clogging; these samples could only be printed to a few layers' thickness (i.e., much thinner than the other printed samples, $0.6 \mathrm{~mm}$ vs. $3.8 \mathrm{~mm}$ ). This difference in thickness might have caused the difference in moduli between the FDM and 
CM specimens. Differences in sample thickness influence edge effects, packing density, and even instrument sensitivity during measurement.

\section{Conclusions}

Carbon fiber-containing ABS resin feedstock at different fiber loadings was prepared, and these feedstock materials were used to successfully fabricate composite specimens by both the FDMprinting and compression-molding processes. The results show that the average fiber length significantly dropped in both processes, likely due to the high-shear mixing step during compounding. While no visible porosity/void was observed in CM samples, significant porosity was observed in FDM-printed samples. With increasing fiber content, voids inside the FDMprinted beads increased, while voids between the beads decreased. FDM-printed samples have high fiber orientation in the printing direction, approaching perfect alignment with the beads. CM samples also show some orientation in the tensile loading direction, probably because of the extrusion process during preform preparation. Samples prepared by both FDM and CM methods show significant increases in both strength and modulus. The higher results obtained with the CM specimens show the dominant effect of porosity on tensile properties over fiber orientation. Furthermore, SEM micrographs show that fibers had pulled out of the matrix, indicating weak interfacial adhesion between the fibers and the matrix.

In summary, this study shows that the FDM process with its controlled orientation and good dispersion capabilities, along with the use of carbon fiber-reinforced feedstock, has great potential for the manufacturing of load-bearing composite parts. Minimizing pore formation during printing and fiber breakage during compounding, as well as improving interfacial 
adhesion between fibers and matrix via surface modification, appear to be the next steps necessary for the FDM process to reach full potential.

\section{Acknowledgements}

This manuscript has been authored by UT-Battelle, LLC, under Contract No. DE-AC0500OR22725 with the US Department of Energy. The US government retains and the publisher, by accepting the article for publication, acknowledges that the US government retains a nonexclusive, paid-up, irrevocable, worldwide license to publish or reproduce the published form of this manuscript, or allow others to do so, for US government purposes.

This research was sponsored by the Laboratory Directed Research and Development Program of Oak Ridge National Laboratory, managed by UT-Battelle, LLC, for the U.S. Department of Energy. Thanks go to the Manufacturing Demonstration Facility at Oak Ridge National Laboratory for the generous use of their facilities and their extremely helpful staff. Additionally, authors would like to thank Mr. John Lindall for his contribution in printing the FDM test samples.

\section{References}

[1] Sood AK, Ohdar RK, Mahapatra SS. Parametric appraisal of mechanical property of fused deposition modelling processed parts. Mater Des 2010;31: 287-95.

[2] Zhong W, Li F, Zhang Z, Song L, Li Z. Short fiber reinforced composites for fused deposition modeling. Mater Sci Eng A 2001; 301: 125-30 .

[3] Fu SY, Lauke B, Mader E, Yue CY, Hu X. Tensile properties of short-glass-fiber- and short-carbon-fiber-reinforced polypropylene composites. Composites Part A 2000; 31: $1117-25$. 
[4] Gray IV RW, Baird DG, Bohn JH. Thermoplastic composites reinforced with long fiber thermotropic liquid crystalline polymers for fused deposition modeling. Polym Compos 1998; 19(4): 383-94.

[5] Es-Said OS, Foyos J, Noorani R, Mandelson M, Marloth R, Pregger BA. Effect of layer orientation on mechanical properties of rapic prototyped samples. Mater Manuf Process 2000; 15(1): 107-22.

[6] Shofner ML, Lozano K, Rodriguez-Macias FJ, Barrera EV. J Appl Polym Sci 2003; 89: 3081-90.

[7] Nikzad M, Masood SH, Sbarski I. Mater Des 2011; 32: 3448-56.

[8] Karsli NG, Aytac A. Tensile and thermomechanical properties of short carbon fiber reinforced polyamide 6 composites. Composites Part B 2013; 61: 270-75.

[9] Botelho EC, Figiel L, Rezende MC, Lauke B. Mehanical behavior of carbon fiber reinforced polyamide composites. Compos Sci Technol 2003; 63: 1843-55.

[10] Tezcan J, Ozcan S, Gurung B, Filip P. Measurement and analytical validation of interfacial bond strength of PAN-fiber-reinforced carbon matrix composites. J Mater Sci 2008; 43(5): 1612-18.

[11] Vautard F, Ozcan S, Poland L, Nardin M, Meyer H. Influence of thermal history on the mechanical properties of carbon-acrylate composites cured by electron beam and thermal processes. Composites Part A 2013; 45: 162-72.

[12] Gordeyev SA, Ferreira JA, Bernardo CA, Ward IM. A promising conductive material: highly oriented polypropylene filled with short vapour-grown carbon fibers. Mater Lett 2001; 51: 32-6. 
[13] Kuriger RJ, Khairul MA, Anderson PD, Jacobson RL. Processing and characterization of aligned vapor grown carbon fiber reinforced polypropylene. Composites Part A 2002; 33: $53-62$.

[14] Fu Sy, Lauke B. Effect of fiber length and fiber orientation distributions on the tensile strength of short-fiber-reinforced polymers. Compos Sci Technol 1996; 56: 1179-90.

[15] Bijsterbosch H, Gaymans RJ. Polyamide 6-long glass fiber injection moldings. Polym Compos 1995; 16: 363-9.

[16] Biolzi L, Castellani L, Pitacco I. On the mechanical response of short fibre reinforced polymer composites. J Mater Sci 1994; 29: 2507-12.

[17] Fu SY, Lauke B. Analysis of mechanical properties of ABS terpolymer reinforced with short glass fibers and calcite particles. J Mater Sci Technol 1997; 13: 389-96.

[18] Ho KC, Hwang JR, Doong JL. Tensile properties of short glass fibre reinforced polycarbonate. Polym Polym Compos 1996; 4: 563-75.

[19] Ramsteiner F, Theysohn R. The influence of fibre diameter on the tensile behavior of short-glass-fibre reinforced polymers. Compos Sci Technol 1985; 24: 231-40.

[20] Sato N, Kurauchi T, Sato S, Kamigaito O. Microfailure behaviour of randomly dispersed short fibre reinforced thermoplastic composites obtained by direct SEM observation. J Mater Sci 1991; 26: 3891-8.

[21] Hine PJ, Davidson N, Duckett RA, Ward IM. Measuring the fibre orientation and modelling the elastic properties of injection-molded long-fibre-reinforced nylon. Comp Sci Technol 1995; 53: 125-31. 
[22] Shiao ML, Nair SV, Garrett PD, Pollard RE. Effect of glass-fibre reinforcement and annealing on microstructure and mechanical behavior of nylon 6,6. Part I. Microstructure and morphology. J Mater Sci 1994; 29: 1973-81.

[23] Shiao ML, Nair SV, Garrett PD, Pollard RE. Effect of glass-fibre reinforcement and annealing on microstructure and mechanical behavior of nylon 6,6. Part II. Mechanical behaviour. J Mater Sci 1994; 29: 1739-52.

[24] Li J, Cai CL. The carbon fiber surface treatment and addition of PA6 on tensile properties of ABS composites. Curr Appl Phys 2011; 11: 50-4.

[25] Rezaei F, Yunus R, Ibrahim NA. Effect of fiberlength on thermomechanical properties of short carbon fiber reinforced polypropylene composites. Mater Des 2009; 30: 260-3.

[26] Li J. Interfacial studies on the $\mathrm{O}_{3}$ modified carbon fiber-reinforced polyamide 6 composites. Appl Surf Sci 2008; 255: 2822-4.

[27] Montes-Moran MA, Hattum FWJ, Nunes JP. A study of the effect of plasma treatment on the interfacial properties of carbon fiber-thermoplastic composites. Carbon 2005; 8: $1795-9$.

[28] ASTM D638, ASTM Standard test method for tensile properties of plastics, in D638-03. 2003, ASTM International: West Conshohocken, PA.

[29] ASTM D4703, ASTM Standard practice for compression molding thermoplastic materials into test specimens, plaques, or sheets, in D4703 - 10a. 2010, ASTM International: West Conshohocken, PA.

[30] Velez-Garcia GM, Wapperom P, Baird DG, Aning AO, Kunc V. Unambiguous orientation in short fiber composites over small sampling area in a center-gated disk, Composites Part A, 2012; 43(1);104-113 
[31] Bay RS, Tucker CL. Stereological measurement and error-estimates for 3-dimensional fiber orientation. Polym Eng Sci 1992; 32(4): 240-53.

[32] Vautard F, Ozcan S, Paulauskas F, Spruiell JE, Meyer H, Lance MJ. Influence of the carbon fiber surface microstructure on the surface chemistry generated by a thermochemical surface treatment. Appl Surf Sci 2012; 261: 473-480.

[33] Vautard F, Grappe H, Ozcan S. Stability of carbon fiber surface functionality at elevated temperatures and it influence on interfacial adhesion. Appl Surf Sci 2013; 268: 61-72.

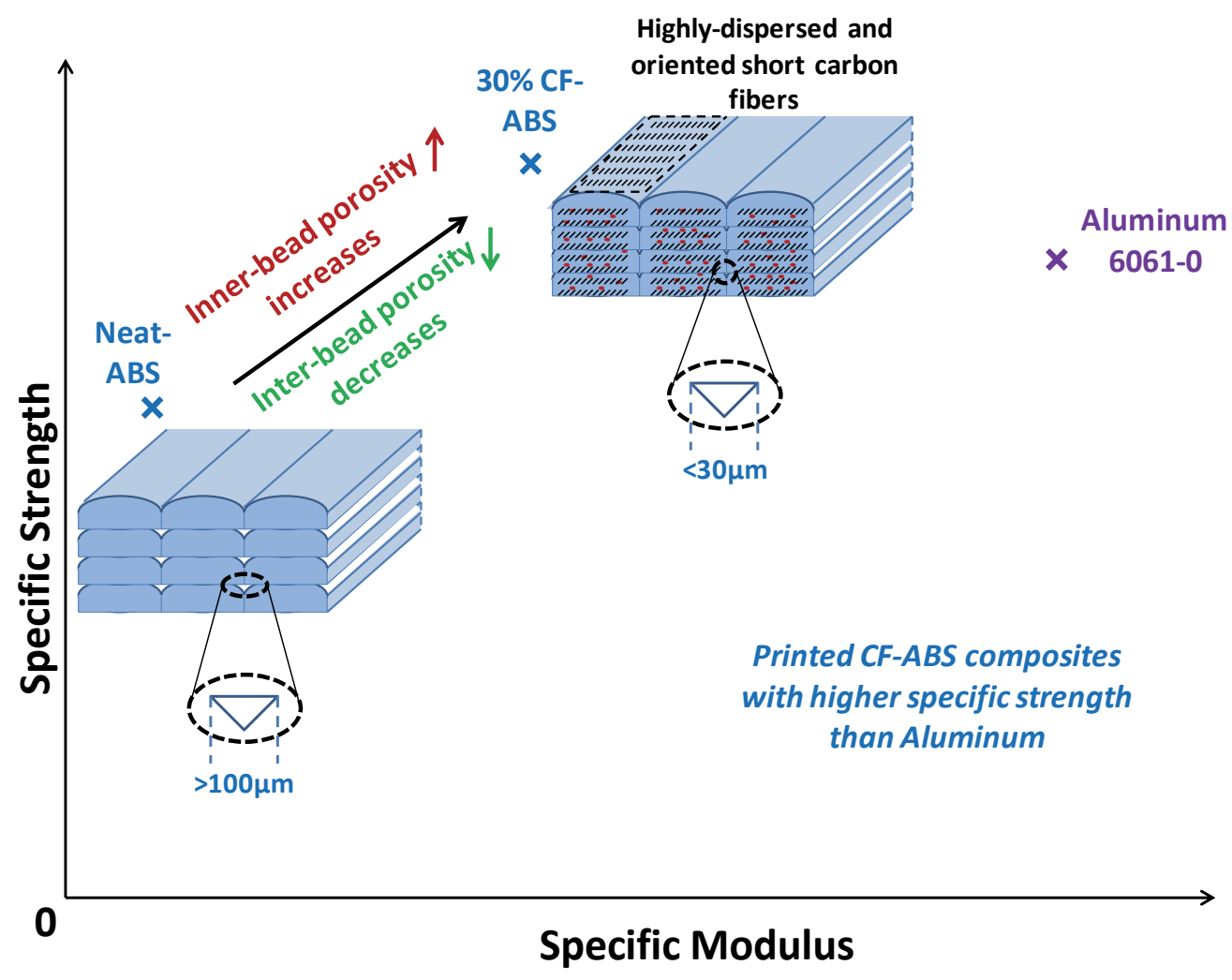

Figure 1. Schematic presentation of 3D-printed fiber-reinforced composite by fused deposition modeling. 

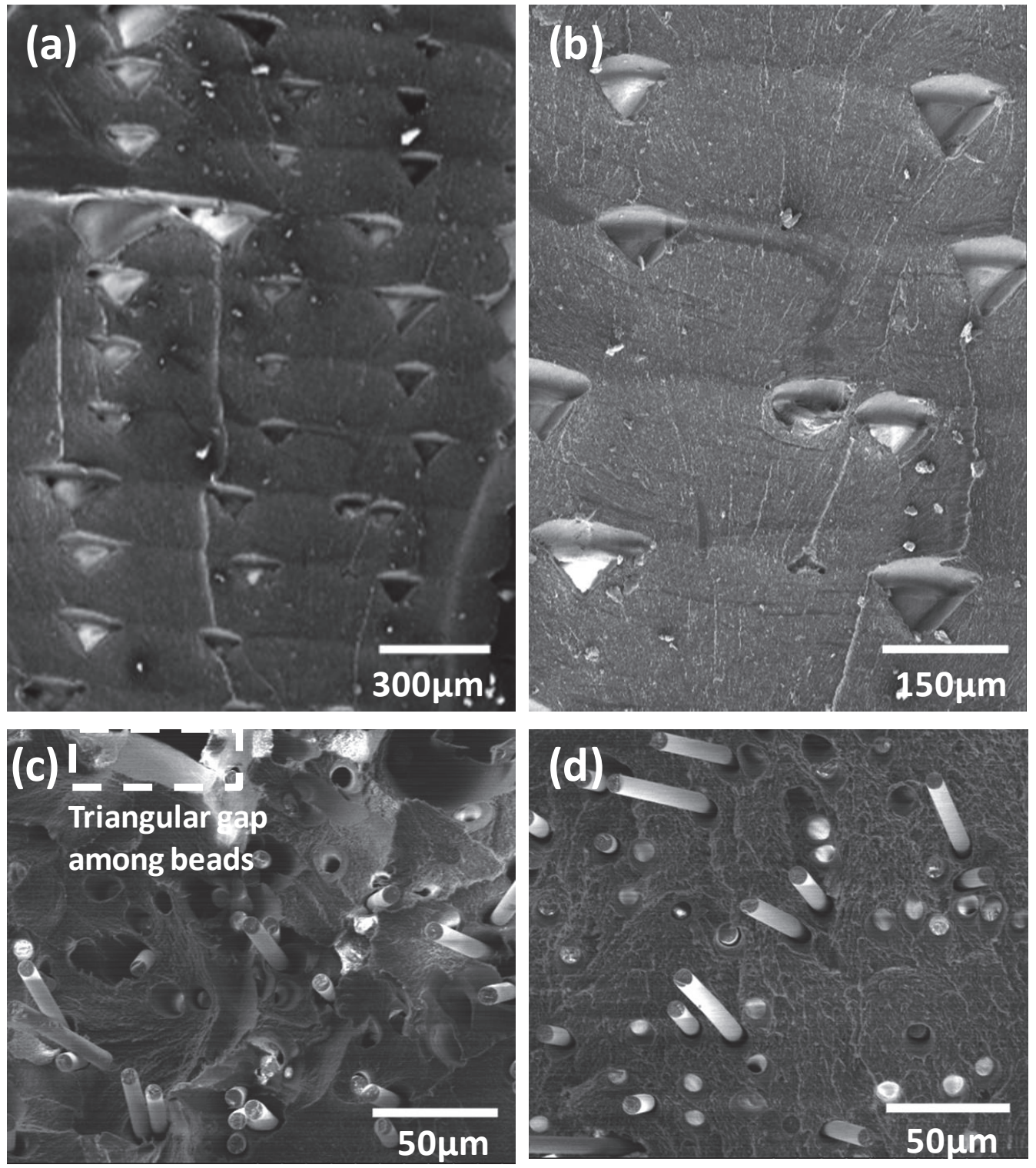

Figure 2. Fracture surface SEM micrographs of (a) and (b) neat-ABS fused deposition modeling (FDM)-printed, (c) $10 \mathrm{wt} \%$ carbon fiber-loaded FDM-printed, and (d) $10 \mathrm{wt} \%$ CF-loaded compression-molded (CM) ABS/CF composites. Protruding fibers are clear of ABS, indicating poor fiber-matrix interfacial adhesion. Pore enlargement is evident around the fibers in the FDM sample, while no significant enlargement is seen in the CM sample. 

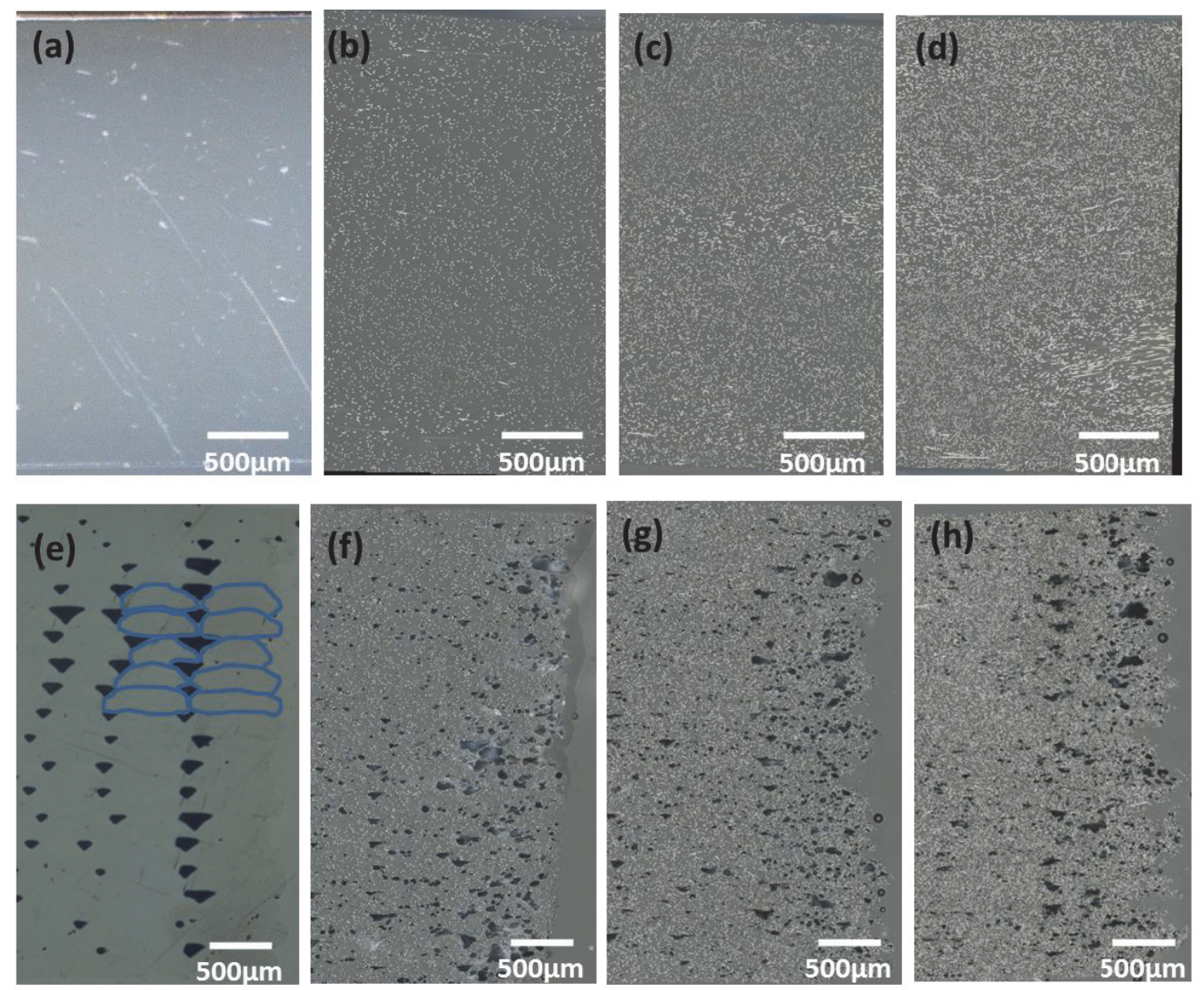

Figure 3. Micrographs of polished surfaces of dog-bone slices. (a) CM neat-ABS, (b) $\mathrm{CM} 10 \% \mathrm{CF}$, (c) $\mathrm{CM} 20 \% \mathrm{CF}$, (d) $\mathrm{CM} 30 \% \mathrm{CF}$, (e) FDM neat-ABS, (f) $\mathrm{FDM} 10 \% \mathrm{CF},(\mathrm{g})$ FDM20\%CF, (h) FDM30\%CF. 

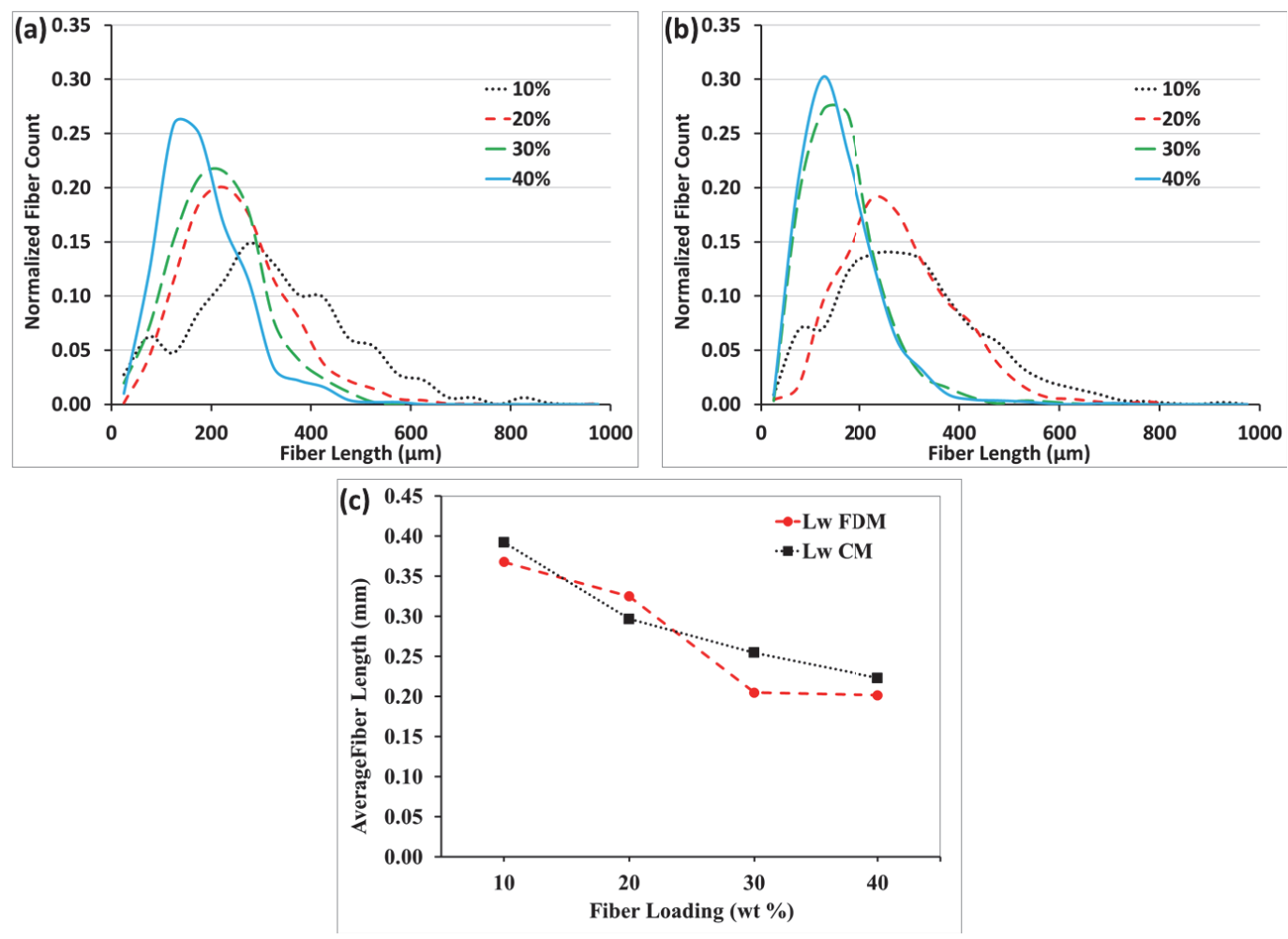

Figure 4. Fiber length distributions ((a) compression-molded, (b) FDM-printed) and (c) weight average fiber lengths of dog-bone samples.

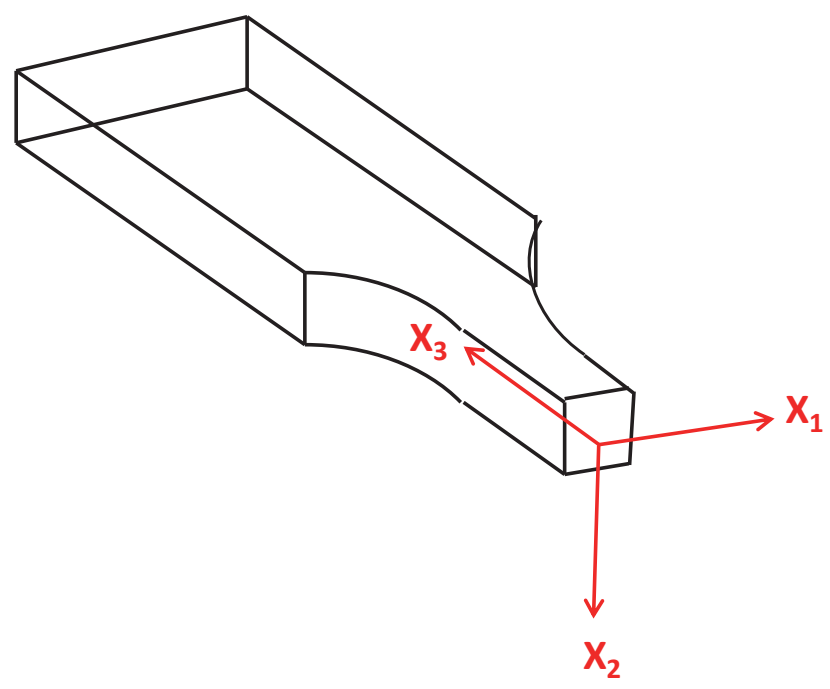

Figure 5. Sketch of a dog-bone sample showing orientation directions. 

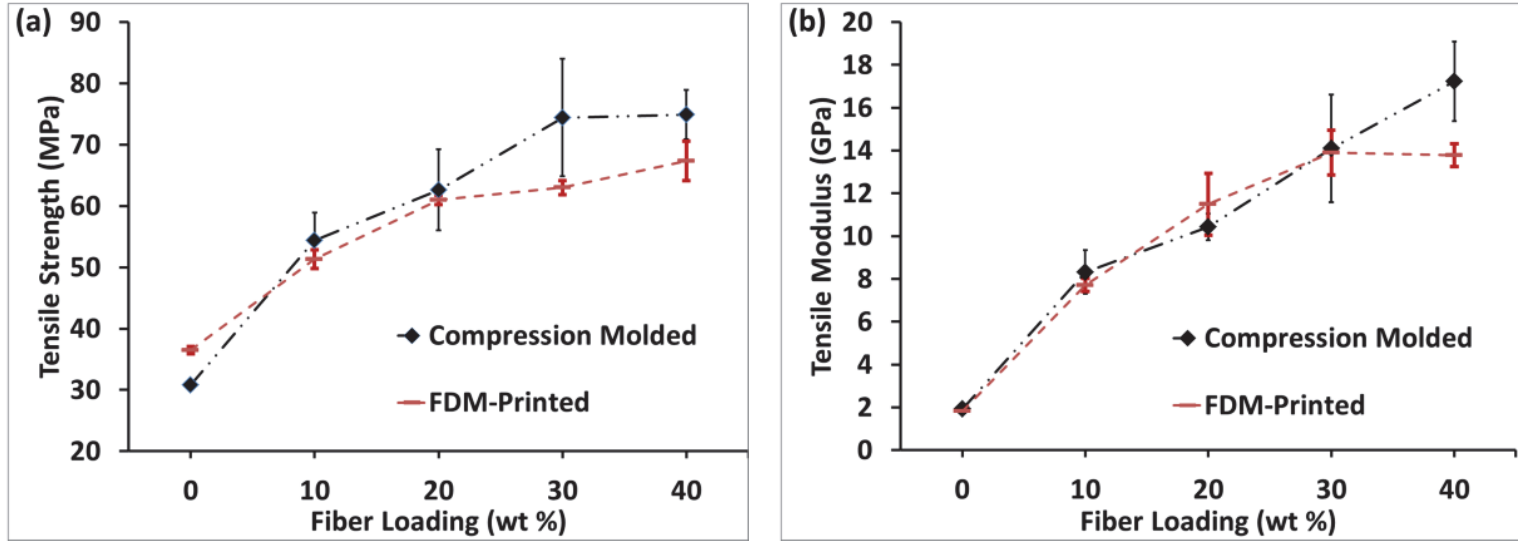

Figure 6. Effect of fiber content and preparation process on (a) tensile strength and (b) modulus, of $\mathrm{ABS} / \mathrm{CF}$ composites. (Each error bar represents one positive and one negative standard deviation. Because the standard deviation for neat-ABS samples were too small, they are not visible in the graph.)

Table 1. Components of the second-order orientation tensor of $\mathrm{ABS} / \mathrm{CF}$ composites.

\begin{tabular}{|c|c|c|c|c|c|c|}
\hline Carbon Fiber (wt\%) & $\mathbf{a}_{11}$ & $\mathbf{a}_{12}$ & $\mathbf{a}_{13}$ & $\mathbf{a}_{22}$ & $\mathbf{a}_{23}$ & $\mathbf{a}_{33}$ \\
\hline \multicolumn{7}{|c|}{ Compression-molded samples } \\
\hline 10 & 0.241 & -0.023 & 0.042 & 0.030 & 0.084 & 0.729 \\
\hline 20 & 0.493 & -0.059 & -0.054 & 0.023 & 0.046 & 0.484 \\
\hline 30 & 0.454 & -0.034 & 0.062 & 0.023 & 0.064 & 0.523 \\
\hline 40 & 0.386 & -0.043 & -0.049 & 0.036 & 0.095 & 0.578 \\
\hline \multicolumn{7}{|l|}{ FDM-printed samples } \\
\hline 10 & 0.055 & 0.005 & 0.038 & 0.030 & 0.127 & 0.915 \\
\hline 20 & 0.064 & 0.004 & 0.024 & 0.028 & 0.121 & 0.909 \\
\hline 30 & 0.060 & -0.002 & -0.006 & 0.039 & 0.143 & 0.901 \\
\hline 40 & 0.093 & -0.005 & -0.018 & 0.038 & 0.139 & 0.869 \\
\hline
\end{tabular}

